\title{
High frequency plant regeneration from mature seed of elite, recalcitrant Malaysian indica rice (Oryza sativa L.) CV. MR 219
}

\begin{abstract}
An efficient in vitro plant regeneration system was established for elite, recalcitrant Malaysian indica rice, Oryza sativa L. CV. MR 219 using mature seeds as explant on Murashige and Skoog and Chu N6 media containing 2,4-dichlorophenoxy acetic acid and kinetin either alone or in different combinations. L-proline, casein hydrolysate and Lglutamine were added to callus induction media for enhancement of embryogenic callus induction. The highest frequency of friable callus induction (84\%) was observed in N6 medium containing $2.5 \mathrm{mg} 1$-1 2,4-dichlorophenoxy acetic acid, $0.2 \mathrm{mg} 1$-1 kinetin, $2.5 \mathrm{mg} 1$ -1 L-proline, $300 \mathrm{mg} 1$-1 casein hydrolysate, $20 \mathrm{mg} 1$-1 L-glutamine and $30 \mathrm{~g} 1$-1 sucrose under culture in continuous lighting conditions. The maximum regeneration frequency $(71 \%)$ was observed, when 30-day-old N6 friable calli were cultured on MS medium supplemented with $3 \mathrm{mg} 1$-1 6-benzyl aminopurine, $1 \mathrm{mg} 1$-1 naphthalene acetic acid, $2.5 \mathrm{mg} 1$-1 L-proline, $300 \mathrm{mg} 1-1$ casein hydrolysate and 3\% maltose. Developed shoots were rooted in half strength MS medium supplemented with $2 \%$ sucrose and were successfully transplanted to soil with $95 \%$ survival. This protocol may be used for other recalcitrant indica rice genotypes and to transfer desirable genes in to Malaysian indica rice cultivar MR219 for crop improvement.
\end{abstract}

Keyword: Callus induction; Oryza sativa ssp. indica; Mature seeds; Plant regeneration; Recalcitrant 